\title{
Externalizing behavior severity in youths with callous- unemotional traits corresponds to patterns of amygdala activity and connectivity during judgments of causing fear
}

\author{
ELISE M. CARDINALE ${ }^{a}$, ANDREW L. BREEDEN ${ }^{a}$, EMILY L. ROBERTSON ${ }^{b}$, LEAH M. \\ LOZIER $^{a}$, JOHN W. VANMETER ${ }^{a}$, ABIGAIL A. MARSH $^{\mathrm{a}}$

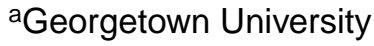 \\ bLouisiana State University
}

\begin{abstract}
Callous-unemotional (CU) traits characterize a subgroup of youths with conduct problems who exhibit low empathy, fearlessness, and elevated externalizing behaviors. The current study examines the role of aberrant amygdala activity and functional connectivity during a socioemotional judgment task in youths with CU traits, and links these deficits to externalizing behaviors. Functional magnetic resonance imaging was used to compare neural responses in 18 healthy youths and 30 youths with conduct problems and varying levels of CU traits as they evaluated the acceptability of causing another person to experience each of several emotions, including fear. Neuroimaging analyses examined blood oxygenation level dependent responses and task-dependent functional connectivity. High-CU youths exhibited left amygdala hypoactivation relative to healthy controls and low-CU youths primarily during evaluations of causing others fear. $\mathrm{CU}$ traits moderated the relationship between externalizing behavior and both amygdala activity and patterns of functional connectivity. The present data suggest that $\mathrm{CU}$ youths' aberrant amygdala activity and connectivity affect how they make judgments about the acceptability of causing others emotional distress, and that these aberrations represent risk factors for externalizing behaviors like rule breaking and aggression. These findings suggest that reducing externalizing behaviors in high-CU youths may require interventions that influence affective sensitivity.
\end{abstract}

\begin{abstract}
Callous-unemotional (CU) traits are the developmental antecedent to adult psychopathy and include shallow affect and reduced empathy and guilt (Frick \& Ellis, 1999). CU traits characterize a subgroup of youths with severe conduct problems (Frick, Ray, Thornton, \& Kahn, 2014; Pardini \& Fite, 2010) whose externalizing behaviors result from distinct patterns of neural dysfunction in the amygdala, prefrontal cortex, and associated regions (Blair, 2013; Viding et al., 2012). The 2015 Diagnostic and Statistical Manual therefore now includes a with limited prosocial emotions specifier indexing CU traits among children with
\end{abstract}

\footnotetext{
Address correspondence : Elise M. Cardinale, Department of Psychology, Georgetown University, 3700 O Street NW, Washington, DC 20057; emc62@georgetown.edu.

Supplementary Material

To view the supplementary material for this article, please visit https://doi.org/10.1017/S0954579417000566.
} 
conduct disorder (Frick et al., 2014), but concerns persist about the difficulty of treating this subgroup (Frick et al., 2014; Hawes, Price, \& Dadds, 2014; Waller, Gardner, \& Hyde, 2013). The development of effective treatments has been impeded in part by limited understanding of the neurobiological mechanisms underlying $\mathrm{CU}$ traits.

Insensitivity to others' distress is a critical feature of CU traits and has been hypothesized to drive externalizing behaviors among high-CU youths. $\mathrm{CU}$ youths exhibit consistent deficits in empathic accuracy, or the ability to recognize fear and sadness communicated by the face, voice, or body (Dawel, O'Kearney, Mckone, \& Palermo, 2012; Marsh \& Blair, 2008) even when these stimuli are presented preattentively (Sylvers, Brennan, \& Lilienfeld, 2011; Viding et al., 2012) or when attention is manipulated (White et al., 2012). These deficits are linked to hypoactivation in the amygdala (Jones, Laurens, Herba, Barker, \& Viding, 2009; Lozier, Cardinale, Vanmeter, \& Marsh, 2014; Marsh et al., 2011; Viding et al., 2012), which has been found to mediate the relationship between $\mathrm{CU}$ traits and externalizing behaviors (Lozier et al., 2014). CU youths' insensitivity to others' fear is paralleled by their reduced personal experiences of fear, including reductions in fear potentiated startle responses (Syngelaki, Fairchild, Moore, Savage, \& van Goozen, 2013), skin conductance responses to threat (Kimonis et al., 2008; Muñoz, Frick, Kimonis, \& Aucoin, 2008), Pavlovian fear conditioning (Fairchild, Stobbe, van Goozen, Calder, \& Goodyer, 2010), and subjectively experienced fear (Jones, Happé, Gilbert, Burnett, \& Viding, 2010; Marsh et al., 2011). Neuroimaging research implicates aberrant structure, function, and connectivity of the amygdala in this reduced fear sensitivity as well (Cohn et al., 2013; De Brito et al., 2011; Finger et al., 2012; Marsh et al., 2008). Together, these findings suggest a neurobiological link between CU youths' muted personal sensitivity to fear and their impaired empathic sensitivity to others' experience of this emotion.

Impaired empathic sensitivity to others' distress, particularly fear, may present a risk factor for the heightened externalizing behaviors these youths exhibit. However, the existing literature is limited in its ability to directly test this link. Previous studies largely assess sensitivity to fear using expressive facial stimuli, which carry two limitations. First, it remains unclear whether amygdala hypoactivity in response to these faces reflects impaired empathic processes or, alternatively, decreased attention to salient perceptual cues, such as the eyes (Dadds, El Masry, Wimalaweera, \& Guastella, 2008). Second, tasks in which participants passively view facial expressions reveal little about cognitive processes that directly drive externalizing behaviors. A hallmark of externalizing behaviors like bullying and aggression is that they cause others fear (Blair, 2005; Nichols, 2001). Thus, a more informative approach would be to measure activity in the amygdala and associated structures while youths make judgments about the ramifications of causing others fear.

Supporting the utility of this approach, recent studies have found that psychopathy in adult community samples is associated with both impairments in identifying behaviors that cause others fear and judgments that causing others fear is relatively more acceptable (Cardinale \& Marsh, 2015; Marsh \& Cardinale, 2012). In these studies, participants read a series of brief statements (e.g., "I could easily hurt you") and judged what emotion each would cause a listener to experience as well as how acceptable it would be to make each statement. Unlike studies measuring responses when passively viewing facial expressions, this paradigm 
allows more direct investigation of the relationship between $\mathrm{CU}$ traits and processes that drive externalizing behaviors, including moral judgments, relevant to the critical question: why do high-CU youths engage in behaviors that cause others fear? Functional neuroimaging results in healthy adults show that whereas low psychopathy scorers preferentially recruit the amygdala and precuneus when performing the emotionally evocative statements task, high psychopathy scorers preferentially recruit the dorsolateral prefrontal cortex, and this pattern of activity is associated with heightened aggression in high psychopathy scorers (Marsh \& Cardinale, 2014).

The current study uses the same paradigm to examine how youths with CU traits reason about causing others fear in order to identify aberrant neural processes during these decisions that may drive increased externalizing behavior. We hypothesized that, relative to healthy youths and low-CU youths with conduct problems, high-CU youths with conduct problems would exhibit dysfunctional patterns of activity and task-dependent functional connectivity in the amygdala when evaluating the acceptability of causing others fear, and that these patterns would correspond to heightened externalizing behaviors in these youths.

\section{Method}

\section{Participants}

Following approval of the protocol by the Georgetown University Institutional Review Board, children ages 10 to 17 were recruited from the Washington, DC, region through advertising, fliers, and referrals seeking children with elevated externalizing behaviors. Eighty-two youths whose families indicated that they exhibit elevated externalizing behaviors completed an initial screening visit to determine eligibility for the scanning portion of the study. Participants were excluded for a full-scale IQ of $<80$ using the Kaufmann Brief Intelligence Test or history of head trauma, neurological disorder, pervasive developmental disorder, nonclinical levels of conduct problems, or magnetic resonance imaging (MRI) contraindications. In addition, no siblings were permitted to participate. Of all 82 youths who completed screening, 30 youths with conduct problems met the criteria for inclusion. Eighteen additional youths were recruited as healthy controls. Additional exclusion criteria for healthy controls included any history of mood, anxiety, or disruptive behavior disorders. All participants were native English speakers. Written informed assent and consent were obtained from children and parents, respectively, before testing.

\section{Clinical measures}

Conduct problems were assessed via parent report on the Strengths and Difficulties Questionnaire (SDQ; Goodman, 1997) and the Child Behavior Checklist (CBCL; Achenbach \& Rescorla, 2000). Youths with clinical-range scores on both the conduct problems subscale of the SDQ (>3) and the externalizing behavior subscale of the CBCL (>98th percentile, age and gender normed) qualified for the conduct problems group (Breeden, Cardinale, Lozier, VanMeter, \& Marsh, 2015; Lozier et al., 2014; Viding et al., 2012). The use of SDQ and CBCL caregiver-report scores to screen for clinical levels of disruptive behavior disorders in community samples is strongly supported by results of a recent systematic review (Warnick, Bracken, \& Kasl, 2008). 
CU traits were assessed using the Inventory of Callous-Unemotional Traits (ICU; Kimonis et al., 2008). Parents and participants completed the ICU separately. Total ICU scores were calculated by summing the highest item rating from either the parent or child version. This method of combining ratings is widely used (Breeden et al., 2015; Gill \& Stickle, 2016; Jones et al., 2009; Kimonis et al., 2016; Lozier et al., 2014; Sebastian et al., 2012; Viding et al., 2012) and follows recommended scoring practices for the parent scale from which the ICU was derived (Frick \& Hare, 2001) because it optimizes accuracy across multiple contexts and reduces susceptibility to social desirability biases (Frick et al., 2003; Piacentini, Cohen, \& Cohen, 1992). The reliability of resulting maximum scores was high (Cronbach a $=0.90$ ). Behavioral and imaging analyses were conducted using two approaches: first, for consistency with previous work (Marsh \& Cardinale, 2012, 2014), group-based analyses of covariance (ANCOVA) following the division of youths with conduct problems into low-CU $(n=15)$ and high-CU $(n=15)$ groups via a median split $(m d n=45.5)$ on ICU scores; and second, regression analyses examining CU traits as a continuous variable (Guay, Ruscio, Knight, \& Hare, 2007; Lozier et al., 2014). Supporting this second approach, a ShapiroWilk test of normality confirms that ICU scores in our sample derive from a normal distribution ( $W=0.97, p=.16$; skewness $=0.06$, kurtosis $=-1.08$ ).

\section{Functional MRI (fMRI) task}

Participants completed an fMRI-adapted version of the Emotionally Evocative Statements Task (EEST; Marsh \& Cardinale, 2014). Across four runs, participants read 120 short ( $M$ word count $=5.89)$ statements, including 20 statements that evoked each of 5 emotions (anger, disgust, fear, happiness, and sadness) plus 20 neutral statements (all statements are available in online-only supplemental set of EEST stimuli). Statements (e.g., fear: "I could easily hurt you") from each emotion category were presented as randomly ordered events for $4000 \mathrm{~ms}$, followed by a 1000-ms fixation. Each run included 10 5000-ms jittered fixation trials and began and ended with a 15000-ms fixation (online-only supplementary Figure S.1). While reading each statement, participants evaluated, "whether it would ever be morally acceptable to make that statement to another person," responding yes or no via button-press with the left or right thumb, respectively. Prior to fMRI testing, all participants were provided extensive verbal instructions and unlimited time for questions, and answered sample questions to ensure complete understanding of the task before testing commenced.

\section{Image acquisition}

Images were acquired using a 12-channel phased-array head coil and 3.0 Tesla Siemens (Erlangen, Germany) TIM Trio. T2* weighted functional images were acquired with a gradient echo-planar imaging sequence (repetition time $[\mathrm{TR}]=2500 \mathrm{~ms}$, echo time $=30 \mathrm{~ms}$, $3.0 \mathrm{~mm}^{3}$ voxels, 46 interleaved slices, matrix $=64 \times 64$, field of view $=192 \mathrm{~mm}$ ). The first four volumes were discarded from each of the four functional runs resulting in 352 total TRs per participant. High-resolution T1-weighted anatomical images were also acquired for each participant $\left(\mathrm{TR}=1900 \mathrm{~ms}\right.$, echo time $=2.52 \mathrm{~ms}, 1.0 \mathrm{~mm}^{3}$ voxels, 176 slices, matrix $=$ $246 \times 256$, field of view $=250 \mathrm{~mm}$ ). 
Image analysis

Functional activity analyses.-Analyses were conducted using Analysis of Functional Neuroimages (AFNI). Data were concatenated, despiked, aligned, normalized, coregistered, and smoothed using a 6-mm Gaussian kernel. Regressors were created for each EEST emotion category, nonresponse trials, and six motion parameters, and convolved with a hemodynamic response function. This resulted in contrasts for each EEST emotion category over baseline for each participant. Anatomical scans were normalized to the Talairach and Tour-noux Atlas. Group-level results were corrected for multiple comparison using Monte Carlo simulation for a corrected alpha of $p=.05$ (66.2 contiguous $3 \times 3 \times 3 \mathrm{~mm}$ voxels at uncorrected $p=.01)$. All results examining amygdala activity were thresholded using small volume correction at $p=.05$ ( 3 contiguous $3 \times 3 \times 3 \mathrm{~mm}$ voxels at uncorrected $p=.01$ ).

Generalized psychophysiological interaction analyses.-Analyses were conducted in SPM8 (Wellcome Trust Department of Cognitive Neurology) using the generalized PPI toolbox (McLaren, Ries, Xu, \& Johnson, 2012). Functional images were slice-time corrected, realigned, coregistered to anatomical scans, normalized to MNI space using parameters calculated during segmentation of anatomical scans, and smoothed using a 6-mm Gaussian kernel. Task-specific functional connectivity with the left amygdala was estimated using generalized psychophysiological interaction analysis (gPPI; McLaren et al., 2012). A gPPI analysis controls for functional connectivity during other task conditions (including baseline), such that the resulting functional connectivity map is specific to the task condition of interest. To avoid biasing gPPI analyses, the amygdala seed region was anatomically defined using the AAL atlas. A design matrix was created for each participant that included stimulus time series for each EEST emotion category, error or nonresponse trials, and six motion parameters, which were convolved with a hemodynamic response function to create psychological regressors. The physiological variable was created through extraction of the deconvolved time series from the left amygdala seed. Again, all group level results were corrected for multiple comparison using Monte Carlo simulation for a corrected alpha of $p$ $=.05(162$ contiguous $2 \times 2 \times 2 \mathrm{~mm}$ voxels at uncorrected $p=.01)$.

\section{Results}

\section{Behavioral responses}

No group differences in psychological or demographic variables, including gender, racial/ ethnic distributions, or age, were observed (Table 1). However, healthy control youths trended younger, $t(31)=1.72, p=.10$, and IQ estimates of high-CU youths were lower than healthy controls, $t(31)=4.24, p<.001$, and low-CU youths, $t(28)=2.27, p=.03$. We therefore included age and IQ as covariates in all analyses.

The proportion of "no" responses to the total number of responses for each emotion category of the EEST was calculated. There were no group differences in total number of responses to the task, $F(45,2)=2.22, p=.12$. A $3 \times 6$ repeated-measures ANCOVA with group (controls, low-CU, high-CU) as a between-subjects factor, emotion as a within-subjects factor, and age and IQ as covariates, found a main effect of emotion, $F(1,215)=7.20, p<.001$. Happinessevoking statements and neutral statements were rated as most acceptable and fear- and 
anger-evoking statements as least acceptable (online-only supplementary Table S.1). No main effect or interaction for group was observed (all $p s>.10$ ). When analyses were repeated with ICU scores entered as a continuous variable, no effect of CU traits was observed (all $p s>.10)$.

\section{Average functional activity}

Whole-brain analysis.-Paralleling our behavioral analyses, we conducted a whole-brain repeated-measures $3 \times 6$ ANCOVA with group as the between-subjects factor and emotion as the within-subjects factor. Age and IQ were included as covariates in this and all subsequent imaging analyses.

The results revealed the hypothesized Group $\times$ Emotion interaction in left amygdala $(x y z=$ $-16,-7,-13, k=5, F=2.84$; Figure 1). To interrogate this interaction, we conducted whole-brain contrast tests within each emotion across our three groups and found group differences only in the left amygdala when participants judged the acceptability of causing fear and anger (Table 2). When high-CU youths judged the acceptability of causing others fear, the left amygdala was less active relative to both healthy controls $(x y z=-10,-1,-22$, $k=26, t=2.71)$ and to low-CU youths $(x y z=-13,-1,-22, k=21, t=3.19)$. When high$\mathrm{CU}$ youths judged the acceptability of causing others anger, the left amygdala ( $x y z=-25$, $-10,-22, k=16, t=2.91$ ) was less active relative to healthy controls, but no differences between high-CU and low-CU youths were observed.

The Group $\times$ Emotion interaction in the left amygdala persisted following the removal of participants with $>15 \%$ of volumes censored due to movement $(x y z=-13,-10,-19, k=6$, $F=3.01$ ), and the exclusion of six youths whose medication could not be withheld prior to scanning ( $x y z=-16,-7,-16, k=12, F=3.63)$. Contrast tests again identified group differences only in the left amygdala during judgments of causing fear. The left amygdala was significantly less active in high-CU youths than in healthy controls $(x y z=-16,-13$, -19 , excluding increased movement: $k=20, t=3.61$, excluding increased movement and medicated: $k=30, t=3.14)$ and low-CU youths $(x y z=-13,-1,-22$, excluding increased movement: $k=33, t=3.16$, excluding increased movement and medicated: $k=42, t=2.80$ ) when they judged the acceptability of causing another person fear (online-only supplementary Figure S.2). Subsequent analyses were conducted using the most stringent inclusion criteria (excluding participants with increased movement or medication, $n=35$; online-only supplementary Table S.2).

Examination of main effects during the task revealed clusters in the left precuneus ( $x y z=$ $-1,-49,44, k=190, F=16.56$ ), right middle frontal gyrus (MFG; $x y z=29,11,53, k=88$, $F=17.35)$, and the left precentral gyrus $(x y z=-55,-10,32, k=73, F=12.96)$. Across all emotion categories of the EEST, high-CU youths showed less activity in the left precuneus relative to both healthy controls $(k=373, t=4.09)$ and low-CU youths $(k=128, t=3.20)$. High-CU youths also showed less right MFG activity relative to low-CU youths $(k=108, t=$ $2.84)$, but not healthy controls, and less left precentral gyrus activity relative to healthy controls $(k=129, t=4.27$ ), but not low-CU youths (see online-only supplementary Table S.3 for follow-up contrasts). 
We found comparable effects when CU traits were included as a continuous predictor of amygdala activity during judgments about causing others fear. To avoid biasing analyses, and to confirm results are significant when activity is confined to the amygdala, we used an anatomically defined left amygdala mask, created using the AFNI Talaraich Atlas, to extract parameter estimates for amygdala blood oxygen level dependent (BOLD) activity during judgments of causing fear. The results of a multiple regression analysis in SPSS with ICU scores entered as a continuous predictor of amygdala BOLD activity, while controlling for age and IQ, confirmed that across all participants $(n=35), \mathrm{CU}$ traits are predictive of decreased amygdala activity when evaluating causing others fear, $t(34)=-2.02, p=.05$. We repeated these analyses including only youths with elevated conduct problems and without medication or increased movement $(n=18)$ and again confirmed that the negative association between $\mathrm{CU}$ traits and amygdala activity, when evaluating causing others fear, persists even within this subset, $t(17)=-2.63, p=.02$.

CU traits, amygdala activity, and externalizing behaviors.-We next examined the relationship between externalizing behaviors and amygdala hypoactivation during judgments of causing others fear. Again, we used an anatomically defined left amygdala mask to extract parameter estimates. Externalizing behavior scores were derived from the externalizing behaviors subscale of the CBCL. The results of a regression analysis found that, when considered in isolation, increased externalizing behaviors predicted decreased amygdala BOLD activity during judgments of causing others fear, while controlling for age and IQ. This effect held both across all youths $(n=35), t(34)=-2.09, p=.05$, and among only youths with elevated conduct problems $(n=18), t(17)=-2.94, p=.01$.

Next, CU traits were examined as a moderator of the relationship between externalizing behaviors and amygdala BOLD activity. In AFNI, we conducted a whole-brain full factorial multiple regression analysis with externalizing behaviors, $\mathrm{CU}$ traits, and the interaction between the two (while controlling for age and IQ) as predictors of amygdala activity during judgments of causing fear (Table 3 ). The results revealed a significant interaction between externalizing behaviors and $\mathrm{CU}$ traits in the left amygdala across all subjects $(n=35 ; x y z=$ $-22,-7,-16, k=10, t=-3.28)$ such that $\mathrm{CU}$ traits significantly moderated the relationship between externalizing behaviors and amygdala activity. As CU traits increased, the relationship between externalizing behaviors and amygdala hypoactivition during judgments of causing fear increased in magnitude. Moreover, the inclusion of the interaction between externalizing behaviors and $\mathrm{CU}$ traits resulted in a significant increase in explained variance of amygdala BOLD activity, $\Delta R^{2}=.57, F(1,29)=14.38, p<.001$, confirming again that $\mathrm{CU}$ traits are a significant moderator of the relationship between externalizing behaviors and amygdala activity during the task, $t(34)=-3.63, p=.001$.

We applied the Johnson-Neyman technique (Johnson \& Neyman, 1936) to identify the ICU score at which the simple slope of amygdala BOLD activity during judgments of causing fear, regressed on externalizing behavior problems, differs from zero. The results revealed that the relationship between externalizing behaviors and reduced amygdala activity was only significant at or above an ICU score of 47.29, $t(34)=-2.05, p=.05$ (Figure 2). Follow-up analyses restricted to only youths with conduct problems $(n=18)$ found nearly identical results. Among these youths, the relationship between externalizing behaviors and 
amygdala BOLD activity was moderated by CU traits, $t(17)=-2.70, p=.02$, with the relationship between externalizing behaviors and amygdala activity only significant at or above an ICU score of $47.84, t(17)=-2.18, p=.05$.

The externalizing subscale of the CBCL comprises three subscales: attention problems, rulebreaking behaviors, and aggression. We examined how $\mathrm{CU}$ traits interact with each of these subscales in predicting amygdala activity using three separate full factorial regressions with each form of externalizing psychopathology, $\mathrm{CU}$ traits, and the interaction between $\mathrm{CU}$ traits and externalizing psychopathology separately predicting amygdala BOLD activity (controlling for age and IQ). Results revealed significant interactions between CU traits and all three subscales: attention problems, $t(34)=-2.95, p=.01$, rule-breaking behaviors, $t$ $(34)=-3.72, p=.001$, and aggression, $t(34)=-3.32, p=.002$. Across all subjects $(n=35)$, as $\mathrm{CU}$ traits increase, the relationship between each externalizing psychopathology and amygdala BOLD hypoactivity increases in magnitude during judgments of causing fear.

\section{Left amygdala functional connectivity}

Generalized psychophysiological interaction analyses.-We evaluated patterns of task-dependent functional connectivity with the left amygdala during judgments of causing others fear. For all connectivity maps resulting from gPPI analyses the left amygdala seed region was anatomically defined using the AAL atlas. In SPM, we conducted a full factorial multiple regression analyses with $\mathrm{CU}$ traits, externalizing behaviors, and the interaction between the two predicting functional connectivity with the left amygdala during judgments of causing fear (controlling for age and IQ). Results revealed that CU traits moderated the relationship between externalizing behaviors and functional connectivity between the left amygdala and several regions, including bilateral thalamus (left $x y z=-12,-24,16, k=172$, $t=4.66$; right $x y z=23,-24,19, k=224, t=4.84)$, right insula $(x y z=27,14,19, k=199, t$ = 3.90), left dorsal lateral prefrontal cortex (dlPFC; $x y z=-35,28,28, k=196, t=3.72)$, left inferior parietal lobule (IPL; $x y z=-33,-36,-31, k=1,376, t=6.45)$, and left MFG $(x y z=$ $-42,-1,21, k=187, t=4.41$; Table 4).

Follow-up univariate general linear model analyses examining the nature of group differences (healthy controls, low-CU youths, and high-CU youths) in functional connectivity with the left amygdala revealed that low-CU youths showed decreased functional connectivity between the left amygdala and bilateral thalamus, left thalamus: $F(2$, $30)=7.23, p=.003$, right thalamus: $F(2,30)=7.66, p=.002$, and right insula: $F(2,30)=$ $6.34, p=.005$, relative to both controls and high-CU youths $(p<.05$ for all pairwise comparisons). No significant differences between controls and high-CU youths for functional connectivity between the left amygdala and bilateral thalamus or right insula were found (all $p \mathrm{~s}>.05$ ). However, high-CU youths showed increased functional connectivity between left amygdala and left dIPFC, $F(2,30)=7.39, p=.002$, left IPL, $F(2,30)=8.66, p$ $=.001$, and left MFG, $F(2,30)=12.84, p<.001$, relative to both controls and low-CU youths ( $p<.05$ for all pairwise comparisons). No significant differences between controls and low-CU youths were found for functional connectivity between the left amygdala and left dlPFC, left MFG, or left IPL (all $p s>.05$; Figure 3). Together, these findings suggest distinct networks of amygdala connectivity emerge in high- and low-CU youths during the 
task that correspond to the increased risk for externalizing behaviors seen in the two subsets of youths.

\section{Discussion}

Our results link severe externalizing behaviors exhibited by high-CU youths to hypoactivation and aberrant functional connectivity in the amygdala during judgments about whether it is acceptable to cause others emotional distress (fear). Relative to both controls and low-CU youths, high-CU youths exhibited left amygdala hypoactivation when they judged whether it would be acceptable to cause another person fear. This pattern persisted whether CU traits were assessed dichotomously or continuously, and whether analyses were conducted across the entire sample, restricted to youths with elevated conduct problems, or restricted to the most stringently controlled sample. Among youths with the highest levels of CU traits, amygdala hypoactivation during judgments of causing fear was associated with a variety of increased externalizing behaviors, including attention problems, rule breaking, and aggression. CU traits also moderated the relationship between externalizing behaviors and functional connectivity with the left amygdala. Low-CU youths showed reduced functional connectivity with the amygdala in regions related to salience and emotion processing, such as the insula and thalamus, whereas high-CU youths showed increased functional connectivity in regions related to cognitive control and semantic reasoning, like the dIPFC, MFG, and IPL. This pattern of results emerged only during judgments about causing fear, but not other emotions. Together, these findings suggest, consistent with previous evidence (Lozier et al., 2014; Marsh et al., 2008; Sylvers et al., 2011; Viding et al., 2012; White et al., 2012), that insensitivity to others' fear in high-CU youths is driven by broad amygdala dysfunction and presents a risk factor for high-CU youths' maladaptive behaviors.

Amygdala hypoactivation and dysfunctional connectivity in high-CU youths and psychopathic adults, while passively viewing fearful facial expressions, may be the single most reliable fMRI finding in this population (Decety, Skelly, Yoder, \& Kiehl, 2014; Dolan \& Fullam, 2009; Jones et al., 2009; Lozier et al., 2014; Marsh et al., 2008; Viding et al., 2012). However, debates persist about the meaning of this finding. One explanation is that fearful expressions represent aversive unconditioned stimuli, and amygdala responses to these expressions in healthy children enable them to learn to avoid behaviors that cause others fear (Blair, 2005). Another possibility is that amygdala responses to fear may reflect the amygdala's role in focusing attention on the most salient low-level perceptual feature of these expressions, the eyes, which aids in recognition of the expressions (Dadds et al., 2008). An alternate (not necessarily mutually exclusive) explanation is that amygdala response to these expressions represents empathic simulation of the target's fearful state, which enables recognition of and appropriate responding to the expression. It is difficult to adjudicate among these possibilities considering only evidence from tasks employing passive viewing of emotional facial expressions.

Because it incorporates verbal stimuli that are neither unconditioned stimuli nor recognizable using low-level perceptual features, the present study provides needed clarity. Our study finds that high-CU youths exhibit amygdala hypoactivation in response not only to the sight of another person's fear, but also to the verbally represented idea of it. 
Understanding that a statement, such as "I could easily hurt you," causes fear relies not on low-level perceptual cues, but on the ability to infer and internally represent another person's emotional state, a low-level and fundamental form of empathy. Our findings are therefore most consistent with the hypothesis that amygdala response to perceived or imagined fear in others may reflect empathic simulation of the target's state and are consistent with the conception of CU traits as fundamentally a disorder of empathy (Blair, 2013). Amygdala hypoactivation in high-CU youths is known to impair their own experiences of fear, including subjective experiences of fear (Jones et al., 2010; Marsh et al., 2011) and physiological responses to fear (Fanti, Panayiotou, Lazarou, Michael, \& Georgiou, 2016). Our findings suggest that amygdala hypoactivation may also impair high-CU youths' ability to internally represent others' fear (Marsh, 2013); in other words, to empathize. These youths' amygdala hypoactivation during the task may therefore help to explain their persistent engagement in behaviors like threats and aggression that cause others fear.

However, additional research linking amygdala dysfunction to behavioral measures of impaired ability to internally represent others' fear is needed to more directly test this theory. Note that unlike previous studies in adults (Cardinale \& Marsh, 2015; Marsh \& Cardinale, 2012, 2014), we did not observe behavioral differences between groups in this paradigm. This may reflect the fact that, unlike adults, youths strongly responded "no" to all four categories of negative statements, which may suggest difficulty making fine-grained distinctions among the various statements in this age group. Heightening our confidence in the task, however, patterns of responses across emotions were similar in youths and previous studies of adults, with both groups evaluating anger- and fear-evoking statements as least acceptable, followed by disgust and sadness, and finally happiness-evoking and neutral statements.

Observed patterns of neural connectivity also suggest the possibility that high- and low-CU youths may use different strategies to arrive at comparable responses during the task. When judging fear-evoking statements, the left amygdala of high-CU youths exhibited increased functional connectivity with left dIPFC, left MFG, and left IPL, while low-CU youths exhibited fear-specific decreased functional connectivity between the left amygdala and bilateral thalamus and right insula. These specific patterns were associated with increased externalizing behaviors within high- and low-CU youths, respectively. Increased functional connectivity between the amygdala and a network of regions implicated in executive functions like cognitive control and semantic reasoning may reflect that, in the absence of robust amygdala BOLD responses, high-CU youths rely more on networks involved in effortful cognitive deliberation during the task, a pattern which may increase their risk for externalizing behaviors in real life. In contrast, decreased functional connectivity between the amygdala with the thalamus and insula may reflect that in low-CU youths, robust amygdala BOLD responses paired with reduced functional connections between the amygdala and emotion processing and salience regions heighten their risk for externalizing behaviors.

One limitation of the current study may be increased movement and use of psychoactive medications that could not be safely withheld in our sample. However, we accounted for this potential concern by repeating key analyses both including and excluding youths with 
increased movement or who were taking medications. The high degree of consistency, regardless of whether these youths were included, increases our confidence both in the robustness of our findings and that these variables were not intrinsically related to the pattern of findings we observed.

Despite these limitations, the present results provide evidence directly linking aberrant amygdala activity and connectivity when judging the acceptability of causing others fear to the heightened externalizing behavior seen in high-CU youths. These results suggest that simply reinforcing abstract awareness of the unacceptability of various externalizing behaviors in high-CU youths may be insufficient to foster behavior changes. Instead, interventions aimed at fostering and enhancing normal patterns of emotional reactivity, with an emphasis on sensitivity to others' internal experiences of distress, may be essential.

\section{Supplementary Material}

Refer to Web version on PubMed Central for supplementary material.

\section{Acknowledgments}

This research was supported by NIH/NICHD Grant R03 HD064906-01, the Georgetown-Howard Universities Center for Clinical and Translational Science (NIH/National Center for Advancing Translational Sciences Grant 1KL2RR031974-01), and the Intellectual and Developmental Disabilities Research Center at Children's National Medical Center (NIH/NICHD Grant 2P30HD040677-11).

\section{References}

Achenbach TM,\& Rescorla L (2000). Child Behavior Checklist. Burlington, VT: University of Vermont.

Blair RJ (2005). Applying a cognitive neuroscience perspective to the disorder of psychopathy. Development and Psychopathology, 17, 865-891. [PubMed: 16262996]

Blair RJ (2013). The neurobiology of psychopathic traits in youths. Nature Reviews Neuroscience, 14, 786-799. [PubMed: 24105343]

Breeden AL, Cardinale EM, Lozier LM, VanMeter JW, \& Marsh AA (2015). Callous-unemotional traits drive reduced white-matter integrity in youths with conduct problems. Psychological Medicine, 45, 3033-3046. [PubMed: 26087816]

Cardinale EM, \& Marsh AA (2015). Impact of psychopathy on moral judgments about causing fear and physical harm. PLOS ONE, 10, e0125708. [PubMed: 25992566]

Cohn MD, Popma A, van den Brink W, Pape LE, Kindt M, van Domburgh L, ... Veltman DJ (2013). Fear conditioning, persistence of disruptive behavior and psychopathic traits: An fMRI study. Translational Psychiatry, 3, e319. [PubMed: 24169638]

Dadds MR, El Masry Y, Wimalaweera S, \& Guastella AJ (2008). Reduced eye gaze explains “fear blindness" in childhood psychopathic traits. Journal of the American Acadamy of Child \& Adolescent Psychiatry, 47, 455-463.

Dawel A, O'Kearney R, McKone E, \& Palermo R (2012). Not just fear and sadness: Meta-analytic evidence of pervasive emotion recognition deficits for facial and vocal expressions in psychopathy. Neuroscience \& Biobehavioral Review, 36, 2288-2304.

De Brito SA, McCrory EJ, Mechelli A, Wilke M, Jones AP, Hodgins S,\& Viding E (2011). Small, but not perfectly formed: Decreased white matter concentration in boys with psychopathic tendencies. Molecular Psychiatry, 16, 476-477. [PubMed: 20548295]

Decety J, Skelly L, Yoder KJ,\& Kiehl KA (2014). Neural processing of dynamic emotional facial expressions in psychopaths. Social Neuroscience, 9, 36-49. [PubMed: 24359488] 
Dolan MC, \& Fullam RS (2009). Psychopathy and functional magnetic resonance imaging blood oxygenation level-dependent responses to emotional faces in violent patients with schizophrenia. Biological Psychiatry, 66, 570-577. [PubMed: 19446795]

Fairchild G, Stobbe Y, van Goozen SH, Calder AJ, \& Goodyer IM (2010). Facial expression recognition, fear conditioning, and startle modulation in female subjects with conduct disorder. Biological Psychiatry, 68, 272-279. [PubMed: 20447616]

Fanti KA, Panayiotou G, Lazarou C, Michael R, \& Georgiou G (2016). The better of two evils? Evidence that children exhibiting continuous conduct problems high or low on callousunemotional traits score on opposite directions on physiological and behavioral measures of fear. Development and Psychopathology, 28, 185-198. [PubMed: 25916948]

Finger EC, Marsh A, Blair KS, Majestic C, Evangelou I, Gupta K, ... Blair RJ (2012). Impaired functional but preserved structural connectivity in limbic white matter tracts in youth with conduct disorder or oppositional defiant disorder plus psychopathic traits. Psychiatry Research: Neuroimaging, 202, 239-244.

Frick PJ, Cornell AH, Bodin DS, Dane HE, Barry CT, \& Loney BR (2003). Callous-unemotional traits and developmental pathways to severe conduct problems. Developmental Psychology, 39, 246260. [PubMed: 12661884]

Frick PJ, \& Ellis M (1999). Callous-unemotional traits and subtypes of conduct disorder. Clinical Child and Family Psychology Review, 2, 149-168. [PubMed: 11227072]

Frick PJ, \& Hare RD (2001). Antisocial Process Screening Device: APSD. Toronto: Multi-Health Systems.

Frick PJ, Ray JV, Thornton LC, \& Kahn RE (2014). Can callous-unemotional traits enhance the understanding, diagnosis, and treatment of serious conduct problems in children and adolescents? A comprehensive review. Psychological Bulletin, 140, 1-57. [PubMed: 23796269]

Gill AD, \& Stickle TR (2016). Affective differences between psychopathy variants and genders in adjudicated youth. Journal of Abnormal Child Psychology, 44, 295-307. [PubMed: 25727716]

Glenn AL, Raine A, Schug RA, Young L, \& Hauser MD (2009). Increased DLPFC activity during moral decision-making in psychopathy. Molecular Psychiatry, 14, 909-911.

Goodman R (1997). The Strengths and Difficulties Questionnaire: A research note. Journal of Child Psychology and Psychiatry, 38, 581-586. [PubMed: 9255702]

Guay JP, Ruscio J, Knight RA, \& Hare RD (2007). A taxometric analysis of the latent structure of psychopathy: Evidence for dimensionality. Journal of Abnormal Psychology, 116, 701-716. [PubMed: 18020717]

Hawes DJ, Price MJ, \& Dadds MR (2014). Callous-unemotional traits and the treatment of conduct problems in childhood and adolescence: A comprehensive review. Clinical Child and Family Psychological Review, 17, 248-267.

Johnson PO, \& Neyman J (1936). Tests of certain linear hypotheses and their application to some educational problems. Statistical Research Memoirs, 1, 57-93.

Jones AP, Happé FG, Gilbert F, Burnett S, \& Viding E (2010). Feeling, caring, knowing: Different types of empathy deficit in boys with psychopathic tendencies and autism spectrum disorder. Journal of Child Psychology and Psychiatry, 51, 1188-1197. [PubMed: 20633070]

Jones AP, Laurens KR, Herba CM, Barker GJ, \& Viding E (2009). Amygdala hypoactivity to fearful faces in boys with conduct problems and callous-unemotional traits. American Journal of Psychiatry, 166, 95-102. [PubMed: 18923070]

Kimonis ER, Fanti KA, Anastassiou-Hadjicharalambous X, Mertan B, Goulter N, \& Katsimicha E (2016). Can callous-unemotional traits be reliably measured in preschoolers. Journal of Abnormal Child Psychology, 44, 625-638. [PubMed: 26344015]

Kimonis ER, Frick PJ, Skeem JL, Marsee MA, Cruise K, Munoz LC, ... Morris AS (2008). Assessing callous-unemotional traits in adolescent offenders: Validation of the Inventory of CallousUnemotional Traits. International Journal ofLaw and Psychiatry, 31, 241-252.

Lozier LM, Cardinale EM, VanMeter JW, \& Marsh AA (2014). Mediation of the relationship between callous-unemotional traits and proactive aggression by amygdala response to fear among children with conduct problems. JAMA Psychiatry, 71, 627-636. [PubMed: 24671141] 
Marsh AA (2013). What can we learn about emotion by studying psychopathy. Frontiers in Human Neuroscience, 7, 181. [PubMed: 23675335]

Marsh AA, \& Blair RJ (2008). Deficits in facial affect recognition among antisocial populations: A meta-analysis. Neuroscience \& Biobehavioral Reviews, 32, 454-465. [PubMed: 17915324]

Marsh AA,\& Cardinale EM (2012). Psychopathy and fear: Specific impairments in judging behaviors that frighten others. Emotion, 12, 892-898. [PubMed: 22309726]

Marsh AA, \& Cardinale EM (2014). When psychopathy impairs moral judgments: Neural responses during judgments about causing fear. Social Cognitive and Affective Neuroscience, 9, 3-11. [PubMed: 22956667]

Marsh AA, Finger EC, Mitchell DG, Reid ME, Sims C, Kosson DS, ... Blair RJ (2008). Reduced amygdala response to fearful expressions in children and adolescents with callous-unemotional traits and disruptive behavior disorders. American Journal of Psychiatry, 165, 712-720. [PubMed: 18281412]

Marsh AA, Finger EC, Schechter JC, Jurkowitz IT, Reid ME, \& Blair RJ (2011). Adolescents with psychopathic traits report reductions in physiological responses to fear. Journal of Child Psychology and Psychiatry, 52, 834-841. [PubMed: 21155775]

McLaren DG, Ries ML, Xu G, \& Johnson SC (2012). A generalized form of context-dependent psychophysiological interactions (gPPI): A comparison to standard approaches. Neuroimage, 61, 1277-1286. [PubMed: 22484411]

Muñoz LC, Frick PJ, Kimonis ER, \& Aucoin KJ (2008). Types of aggression, responsiveness to provocation, and callous-unemotional traits in detained adolescents. Journal of Abnormal Child Psychology, 36, 15-28. [PubMed: 17882544]

Nichols S (2001). Mindreading and the cognitive architecture underlying altruistic motivation. Journal of the American Academy of Child\&Adolescent Psychiatry, 48, 554-562.

Pardini D, \& Fite P (2010). Symptoms of conduct disorder, oppositional defiant disorder, attentiondeficit/hyperactivity disorder, and callous-unemotional traits as unique predictors of psychosocial maladjustment in boys: Advancing an evidence base for DSM-V. Journal of the American Academy of Child \& Adolescent Psychiatry, 49, 1134-1144. [PubMed: 20970701]

Piacentini JC, Cohen P, \& Cohen J (1992). Combining discrepant diagnostic information from multiple sources: Are complex algorithms better than simple ones. Journal of Abnormal Child Psychology, 20, 51-63. [PubMed: 1548394]

Sebastian CL, McCrory EJ, Cecil CA, Lockwood PL, De Brito SA, Fontaine NM, \& Viding E (2012). Neural responses to affective and cognitive theory of mind in children with conduct problems and varying levels of callous-unemotional traits. Archives of General Psychiatry, 69, 814-822. [PubMed: 22868935]

Sylvers PD, Brennan PA, \& Lilienfeld SO (2011). Psychopathic traits and preattentive threat processing in children: A novel test of the fearlessness hypothesis. Psychological Science, 22, 1280-1287. [PubMed: 21881061]

Syngelaki EM, Fairchild G, Moore SC, Savage JC, \& van Goozen SH (2013). Affective startle potentiation in juvenile offenders: The role of conduct problems and psychopathic traits. Social Neuroscience, 8,112-121. [PubMed: 22856454]

Viding E, Sebastian CL, Dadds MR, Lockwood PL, Cecil CA, De Brito SA,\& McCrory EJ (2012). Amygdala response to preattentive masked fear in children with conduct problems: The role of callous-unemotional traits. American Journal of Psychiatry, 169, 1109-1116. [PubMed: 23032389]

Waller R, Gardner F, \& Hyde LW (2013). What are the associations between parenting, callousunemotional traits, and antisocial behavior in youth? A systematic review of evidence. Clinical Psychology Review, 33, 593-608. [PubMed: 23583974]

Warnick EM, Bracken MB, \& Kasl S (2008). Screening efficiency of the Child Behavior Checklist and Strengths and Difficulties Questionnaire: A systematic review. Child and Adolescent Mental Health, 13, 140-147.

White SF, Marsh AA, Fowler KA, Schechter JC, Adalio C, Pope K, ... Blair RJ (2012). Reduced amygdala response in youths with disruptive behavior disorders and psychopathic traits: Decreased emotional response versus increased top-down attention to nonemotional features. American Journal of Psychiatry, 169, 750-758. [PubMed: 22456823] 

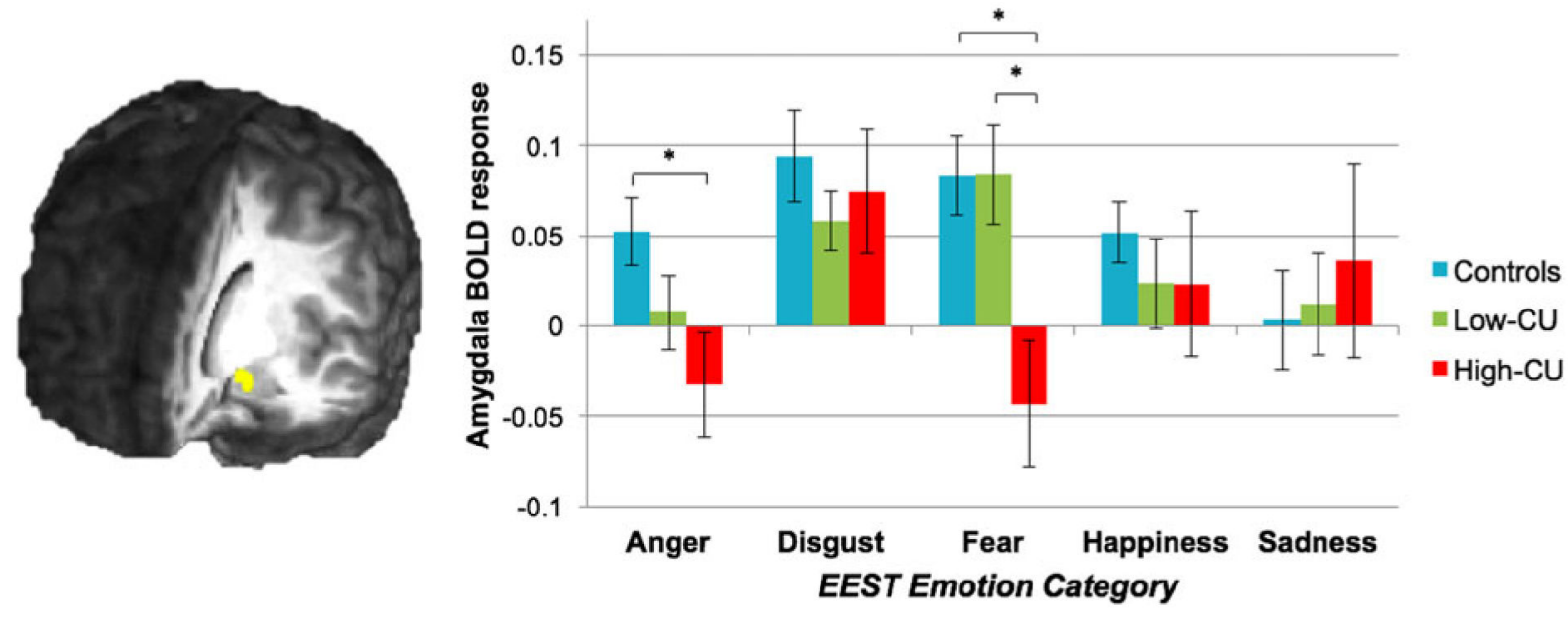

Figure 1.

(Color online) The results of a repeated measures analysis of covariance $(N=48)$ found a significant Group $\times$ Emotion interaction in the left amygdala such that during judgments of the permissibility of causing others' fear, high callous-unemotional (CU) youths show decreased amygdala activity in comparison to both healthy controls (HC) and low-CU youths. Error bars represent the standard error. BOLD, blood oxygen level dependent; EEST, Emotionally Evocative Statements Task. 


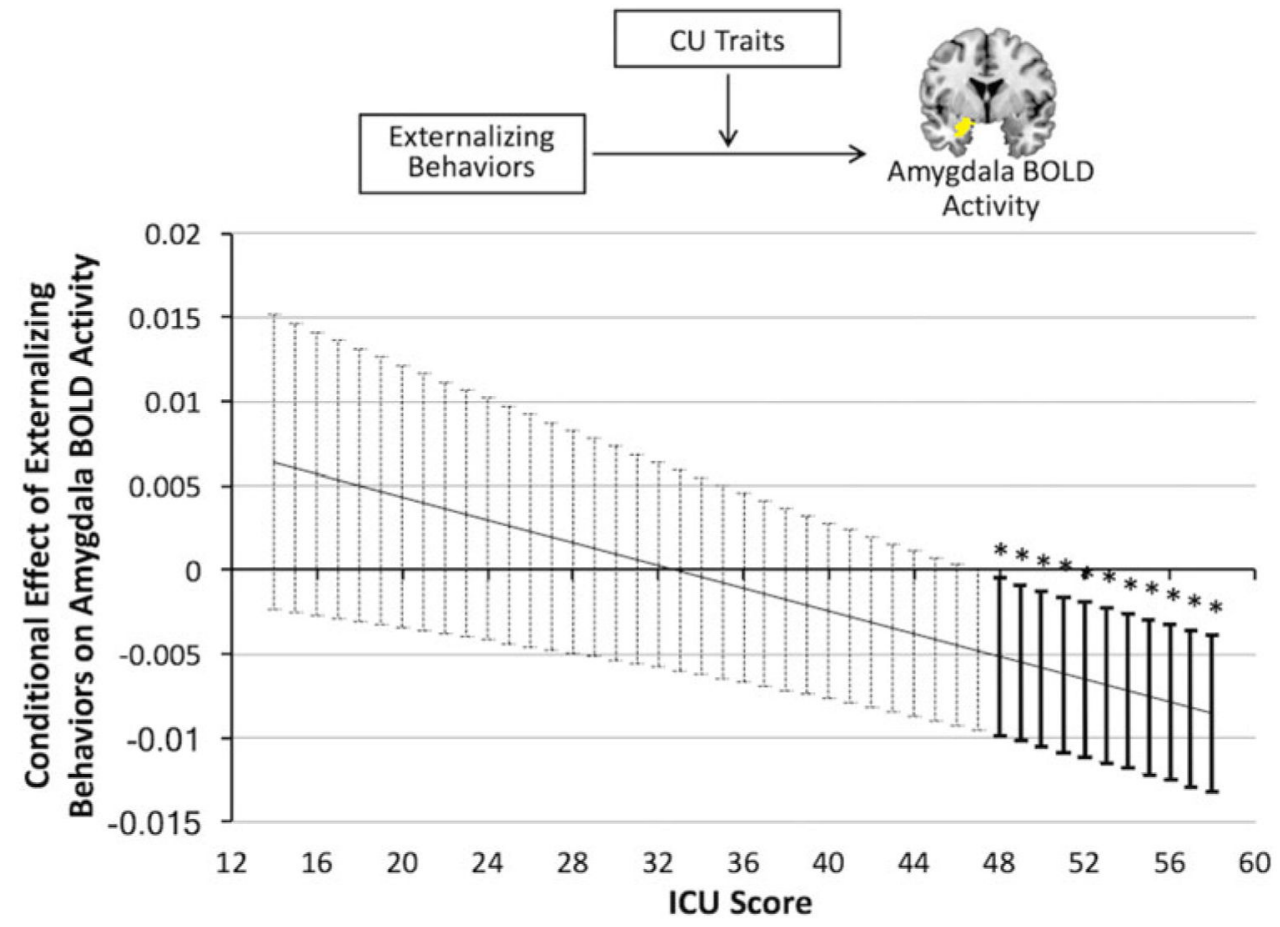

Figure 2.

(Color online) The results of a multiple regression analysis, in a sample of 35 youths free of movement issues and medication during the time of the scan, show that callous-unemotional (CU) traits moderate the relationship between externalizing behavior and amygdala activity during judgments about causing fear in others such that the conditional effect of externalizing behaviors on amygdala activity is only significant at scores $>47.84$ on the Inventory of Callous-Unemotional Traits (ICU). Error bars represent 95\% confidence intervals for the estimated simple slope at each score on the ICU. BOLD, blood oxygen level dependent. 
(a)
Low-CU < HC \& High-CU

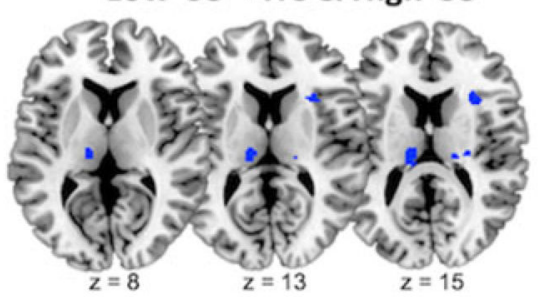

(b)

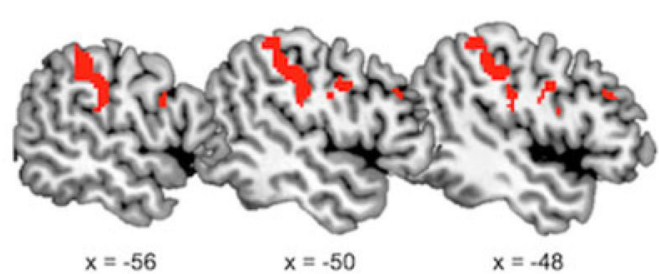

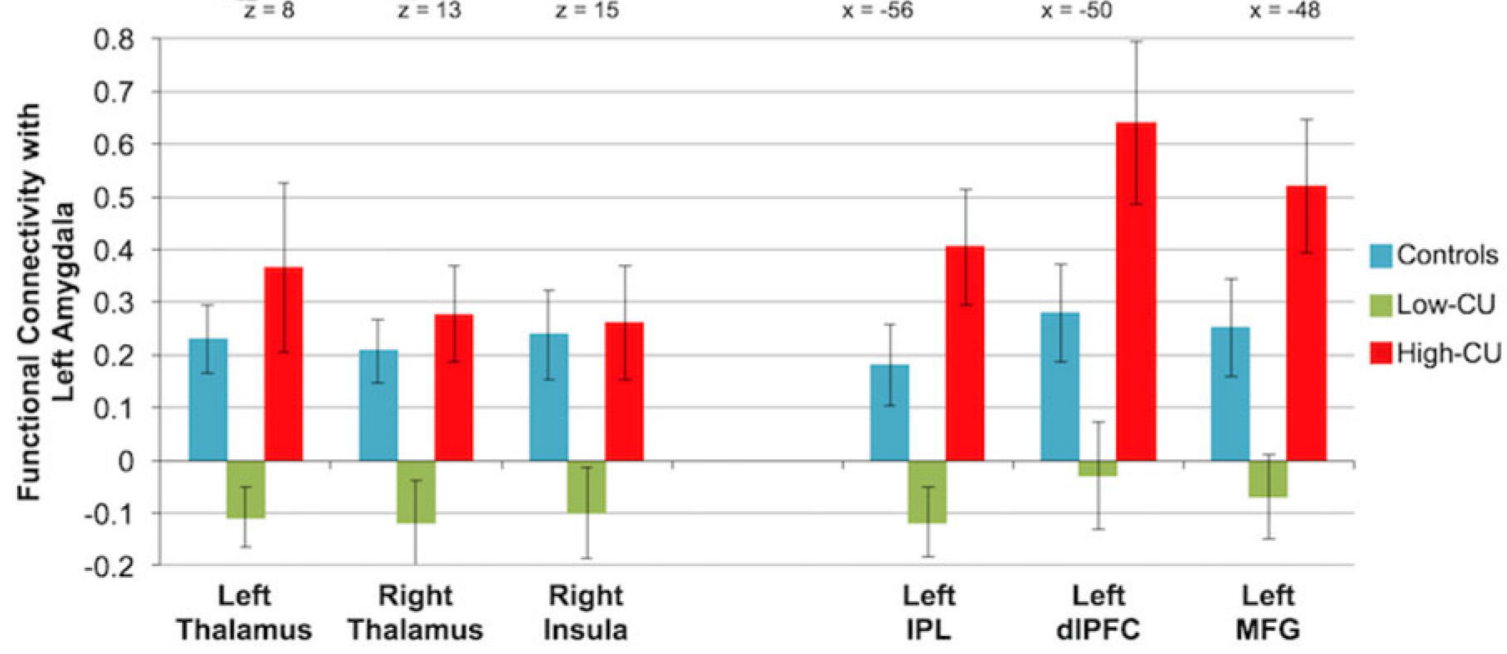

Figure 3.

(Color online) The results from a multiple regression analysis in a sample of 35 youths free of movement issues and medication at time of the scan found that callous-unemotional (CU) traits moderated the relationship between externalizing behaviors and functional connectivity between the left amygdala such that increased externalizing behaviors are associated with (a) decreased functional connectivity between the left amygdala with bilateral thalamus and right insula and in low-CU youths and (b) increased functional connectivity between left amygdala and left dorsal lateral prefrontal cortex (dIPFC), left middle frontal gyrus (MFG), and left inferior parietal lobule (IPL) in high-CU youths. Error bars represent the standard error. HC, healthy controls. 


\section{를




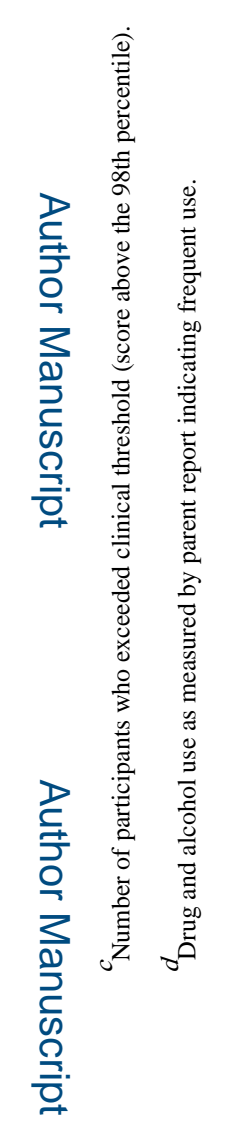

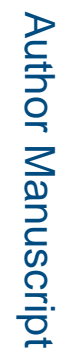

를

Dev Psychopathol. Author manuscript; available in PMC 2020 March 04. 


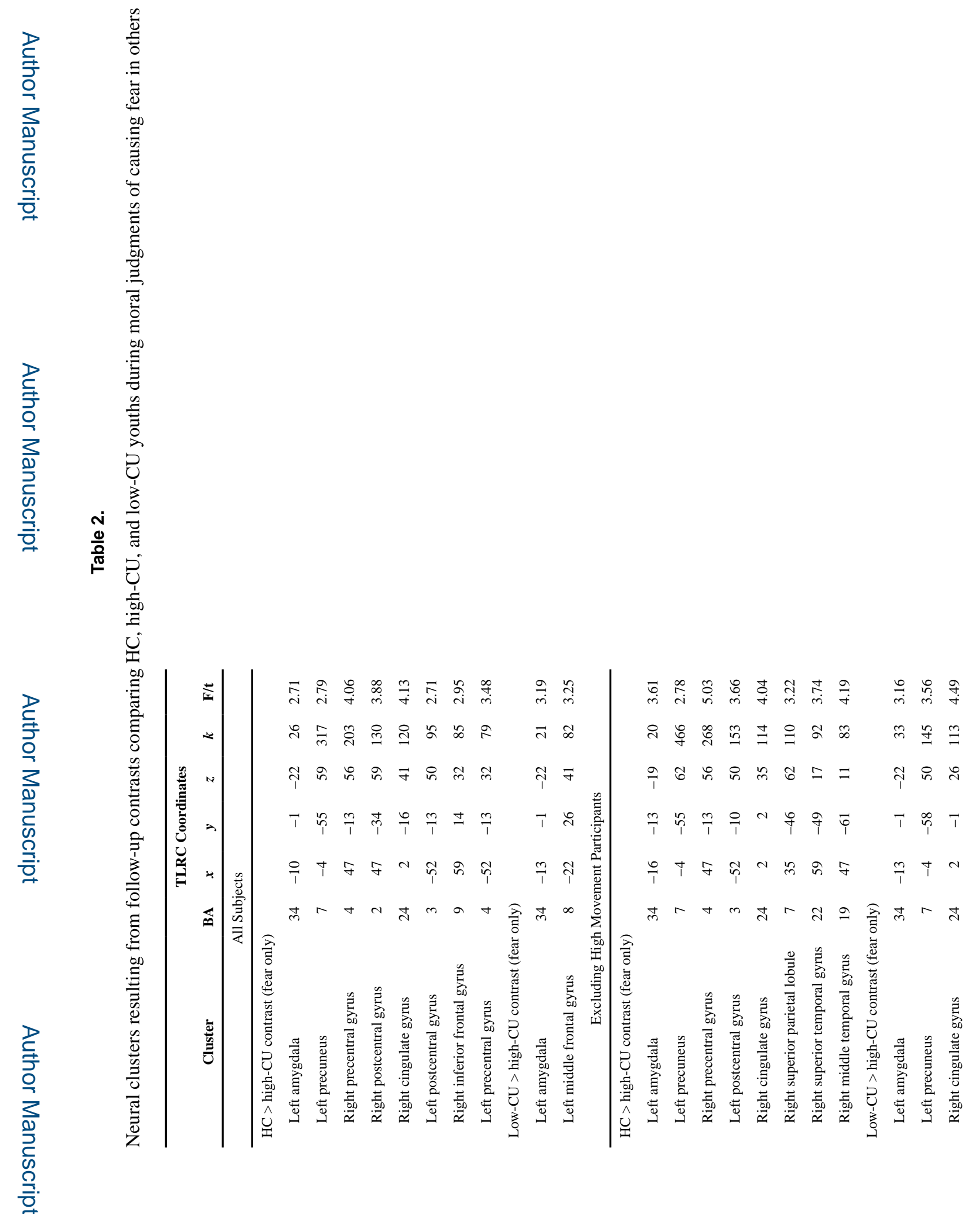

Dev Psychopathol. Author manuscript; available in PMC 2020 March 04. 


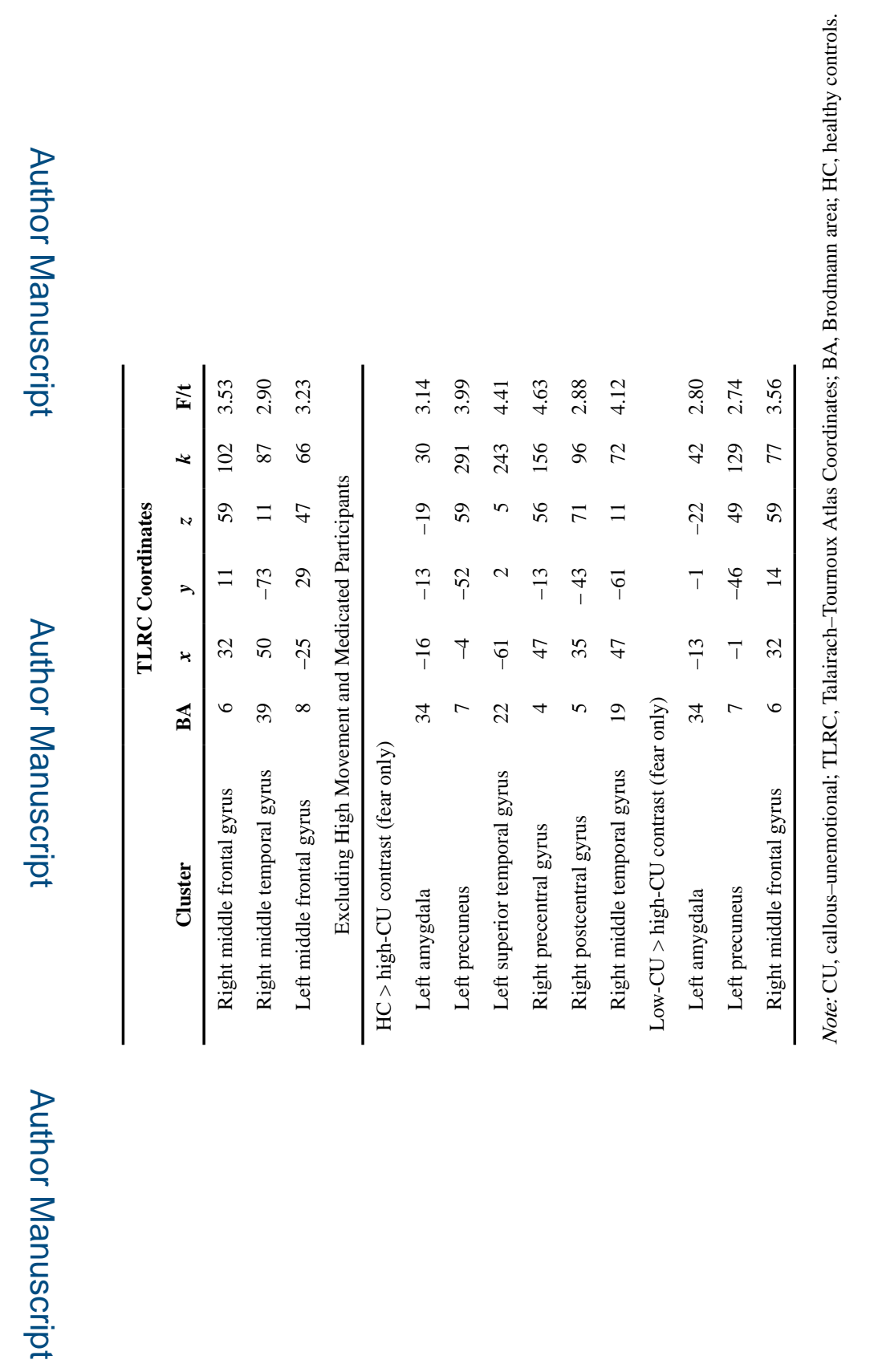

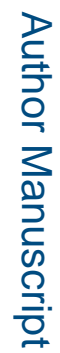

Dev Psychopathol. Author manuscript; available in PMC 2020 March 04. 


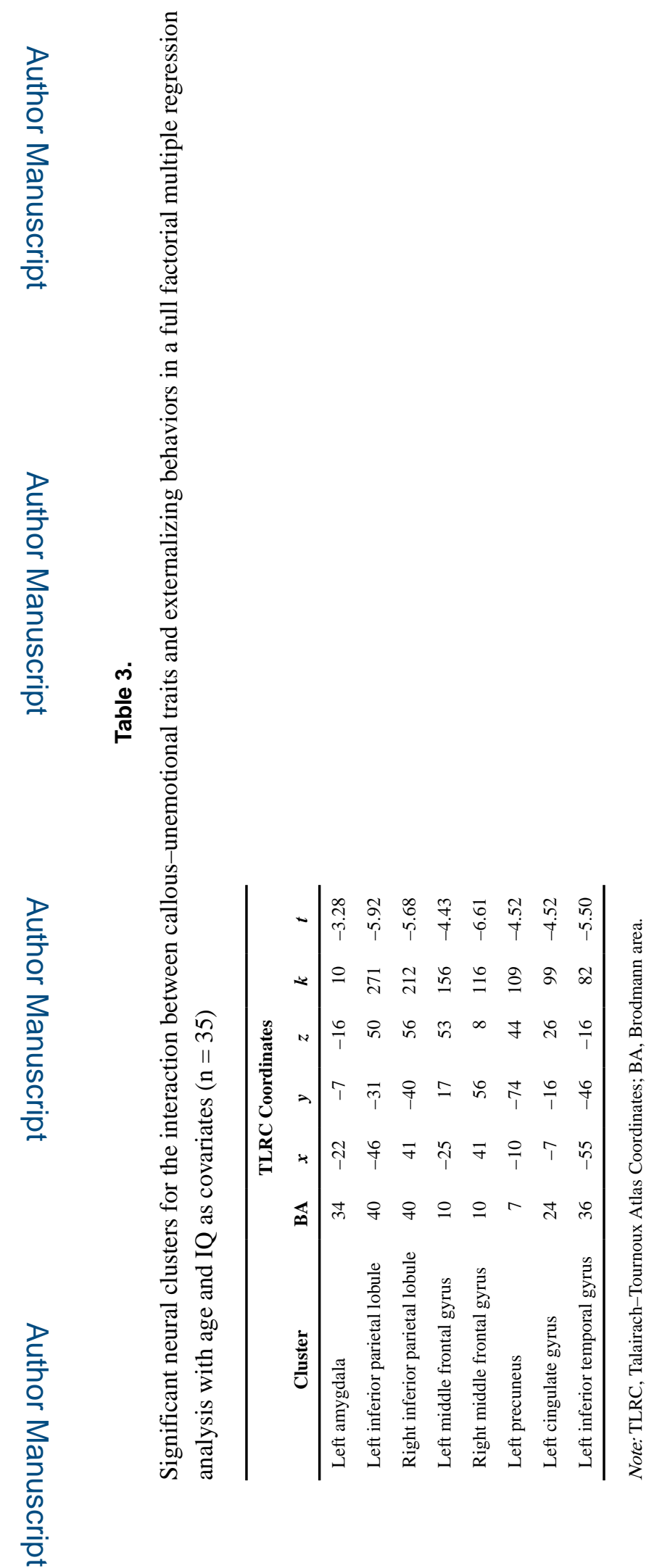

Dev Psychopathol. Author manuscript; available in PMC 2020 March 04. 


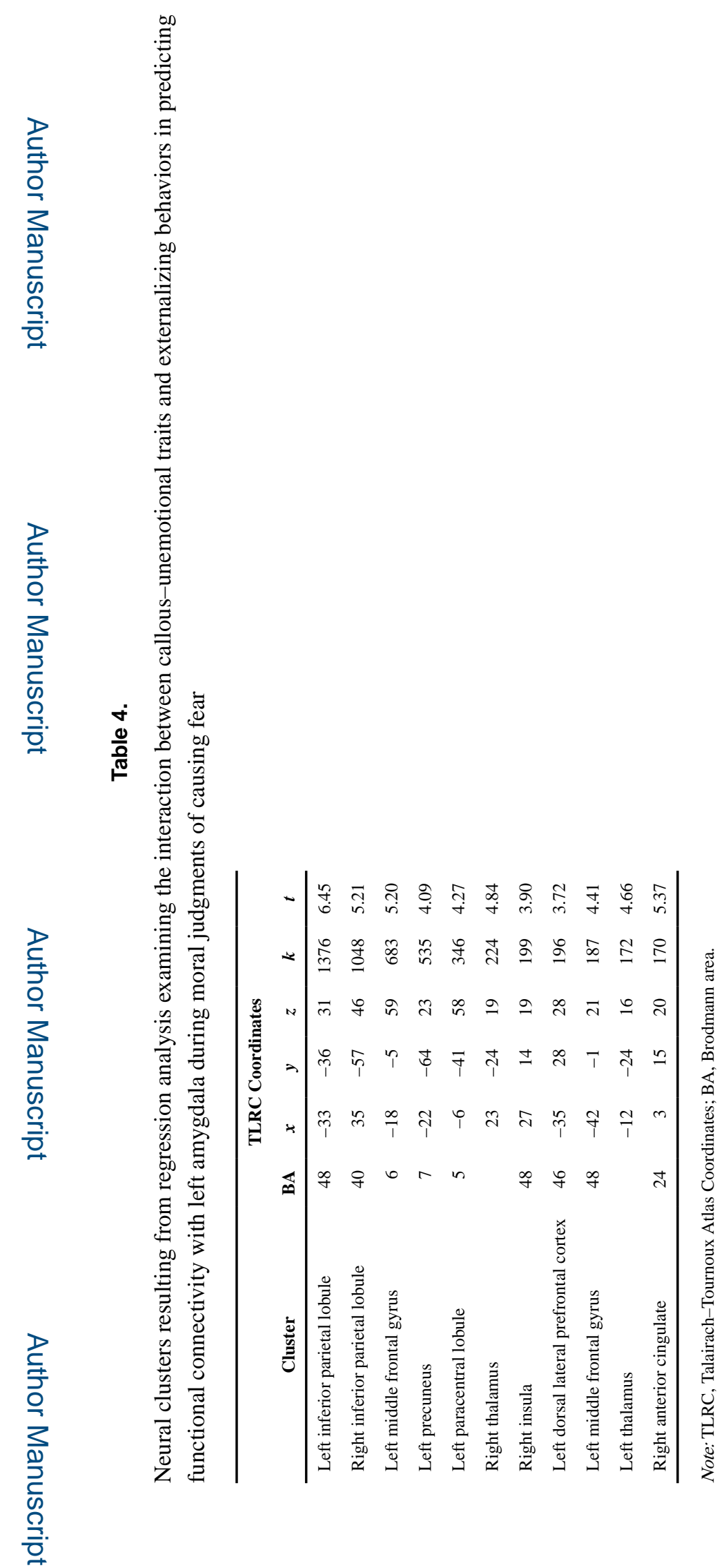

Dev Psychopathol. Author manuscript; available in PMC 2020 March 04. 